\title{
Microstrain and texture in rotary swaged W-Ni-Co pseudoalloy
}

\author{
P. Strunz ${ }^{1}$, R. Kocich², P. Beran ${ }^{1,3}$, L. Kunčická2 ${ }^{2}$ D. Canelo-Yubero ${ }^{1}$, Ch. Hervoches ${ }^{1}$ \\ ${ }^{I}$ Nuclear Physics Institute of the CAS, 25068 Řež, Czech Republic, \\ ${ }^{2}$ Faculty of Materials Science and Technology, VŠB-Technical University of Ostrava, 70800 Ostrava-Poruba, Czech Republic, \\ ${ }^{3}$ European Spallation Source ERIC, 22592 Lund, Sweden \\ strunz@ujf.cas.cz
}

Due to their excellent mechanical properties and high specific mass, tungsten heavy alloys are used in demanding applications, such as kinetic penetrators, gyroscope rotors, or radiation shielding [1]. However, their composite structure, consisting of hard tungsten particles embedded in a soft matrix [2], makes the deformation processing a challenging task. This study focused on the characterization of deformation behaviour during thermomechanical processing of a W-Ni-Co tungsten heavy alloy (produced by powder metallurgy) via the method of rotary swaging (aimed at still improving properties) at ambient temperature and at $900^{\circ} \mathrm{C}$.

Swaging changed mechanical properties, and - as an important step to optimize microstructure and mechanical properties is to understand the underlying processes - the aim of the neutron diffraction study was to determine texture and to characterize microstrain, dislocations as well as the active slip system. The strength of neutron diffraction method lies in the information provision from the bulk of the sample, i.e. not only from its near-surface region. This advantage is still amplified for materials with very high Xray absorption (like tungsten alloys) and/or with large grains.

First, phase identification was done from the diffraction patterns. The detected main phase was corresponding to the original tungsten powder grains of bcc structure, the second (in fact matrix) phase, was Ni-Co solid solution with fcc structure [3]. Peak broadening after swaging was visible in the soft matrix phase.

Further, texture measurement using neutron diffraction was done, which shows that the original as sintered material had for the tungsten phase no texture. It also shows that there were very large grains of Ni-Co matrix phase in the as sintered bar, without any clear preferential orientation. During rotary swaging, the large grains of Ni-Co are fractioned to fine-grained microstructure. A strong texture formed in both phases after rotary swaging [4]. Both bcc phase and fcc phase, after rotary swaging, have the same texture type as for wire drawing. It can be thus concluded that the primary deformation mechanism for rotary swaging was the same as for wire drawing. The textures for cold and hot swaging are qualitatively the same, but stronger for cold swaging which indicates that secondary deformation mechanisms are also active for the hot swaging. The deformation was also connected with formation of residual macrostresses [4,5].

The peak broadening was evaluated for the neutron-diffraction peaks of the relatively soft Ni-Co matrix phase [3]. The modified Williamson-Hall plot shows that the microstrain increased approximately 3 times after rotary swaging. In accord with the texture measurement, the edge dislocations with $<110>\{111\}$ slip system (typical in fcc) provide such contrast factor, that the integral breaths of the individual reflections fit very well to straight lines. Interesting is the Ni-Co matrix in non-deformed as-sintered bar where the contrast factor for screw $<111>$ dislocation fits best with the measured integral breaths. The dislocation densities from the slope of the modified Williamson-Hall plot were estimated. The dislocation density increased approximately 5 times after rotary swaging, which is linked with the mechanical properties: swaged samples exhibited substantial strengthening - primarily caused by the increase in dislocation density. Further, the dislocation density is $15 \%$ higher for the sample swaged at room temperature than for the sample deformed at $900^{\circ} \mathrm{C}$, which fits the trend observed in stress-strain curve.

[1] Kocich, R.; Kunčická, L.; Dohnalík, D.; Macháčková, A. \& Šofer, M. (2016) Int. J. Refract. Met. Hard Mater. 61, $264-272$.

[2] Durlu, N.; Caliskan, N.K. \& Sakir, B. (2014) Int. J. Refract. Met. Hard Mater 42, 126-131.

[3] Strunz, P.; Kunčická, L.; Beran, P.; Kocich, R. \& Hervoches, C. (2020). Materials 13, 208, doi:10.3390/ma13010208

[4] Strunz, P.; Kocich, R.; Canelo-Yubero, D; Macháčková A.; Beran, P. \& Krátká, L. (2020) Materials 13, 2869; doi:10.3390/ma13122869

[5] Canelo-Yubero, D.; Kocich, R.; Hervoches, Ch.; Strunz, P.; Kunčická, L. \& Krátká, L. (2021) Metals and Materials International, https://doi.org/10.1007/s12540-020-00963-8

Keywords: tungsten heavy alloys; rotary swaging; neutron diffraction; dislocations; microstrain; texture

The authors acknowledge support for this research by Czech Science Foundation, grant No. 19-15479S. Measurements were carried out at the CANAM infrastructure of the NPI CAS Řež. The presented results were obtained also with the use of infrastructure Reactors LVR-15 and LR-0 financially supported by the Ministry of Education, Youth and Sports - project LM2018120.

Acta Cryst. (2021), A77, C847 